\title{
The development of Electro-hydraulic servo control system for milling based on ARM
}

\author{
Liping $\mathrm{Xu}^{1, \mathrm{a}}$, Ke Liang${ }^{2, \mathrm{~b}}$, Dezhi Ren ${ }^{3, \mathrm{c}}$ \\ 1,2,3 Henan University of Science \& Technology \\ School of Mechatronica Engineering \\ Luoyang ,China \\ axlpzz@163.com, bkk053@sina.com, 'renthree2000@163.com
}

\begin{abstract}
To overcome the long servo cycle, poor stability, weak parameter adaptability and other poor performances of domestic developed electro-hydraulic servo system of milling, it adopts the embedded design to set up electro-hydraulic servo control system based on STM32 MCU to realize precise control of copper tube surface processing. After an overall design of control system was proposed, the hardware design based on STM32 MCU is expatiated, and a complex control algorithm is designed based on position control and force control. Field tests indicate that the designed electrohydraulic servo control system is worthy to popularize with its high precision, fast response, stable performance, and the quality and efficiency of copper tube processing have been improved.
\end{abstract}

Keywords-Electro-hydraulic servo control system, STM32, Position control , Force control

\section{INTRODUCTION}

Milling machine is an critical equipment for milling oxide layer surface of copper tube. As electro-hydraulic servo system widely used in the milling machine, as well as the increasing requirements on milling speed and quality, the requirements for milling machine control system is also getting higher. Currently, although the imported scalping machine milling control system is excellent in performance, it gets shortcomings like high price, tough to grasp the critical control technology, and poor compatible with the domestic milling equipment[1]. The domestic developed milling machine control systems mainly adopt general control system like PLC, with big problems in suitability, accuracy and efficiency. To solve these problems, this article introduces the modern EDA adopted mlling machine control system, with ARM Cortex-M3 core and STM32F107RCT6 control nucleus, and a composite control arithmetic of position control and force control. Then an embedded milling machine control system applicable design with higher performance, lower cost and lower dissipation is provided.

\section{SYSTEM ANALYSIS OF WORKING PRINCIPLE}

As shown in Figure1. The power part of milling machine consists of arc milling cutter, slider, wheel, servo hydraulic cylinder, servo valve body. During the milling process, the copper tube enters on the left side, with a certain speed gets through the cutter for milling. Two sets of power mill two surfaces simultaneously in a milling process. Then, roll the copper pipe to mill the other two surfaces. A milling process for a copper tube can be completed with three rotations. The copper tube is fixed by clamping support mechanism to ensure it cannot rotate freely during the milling process.

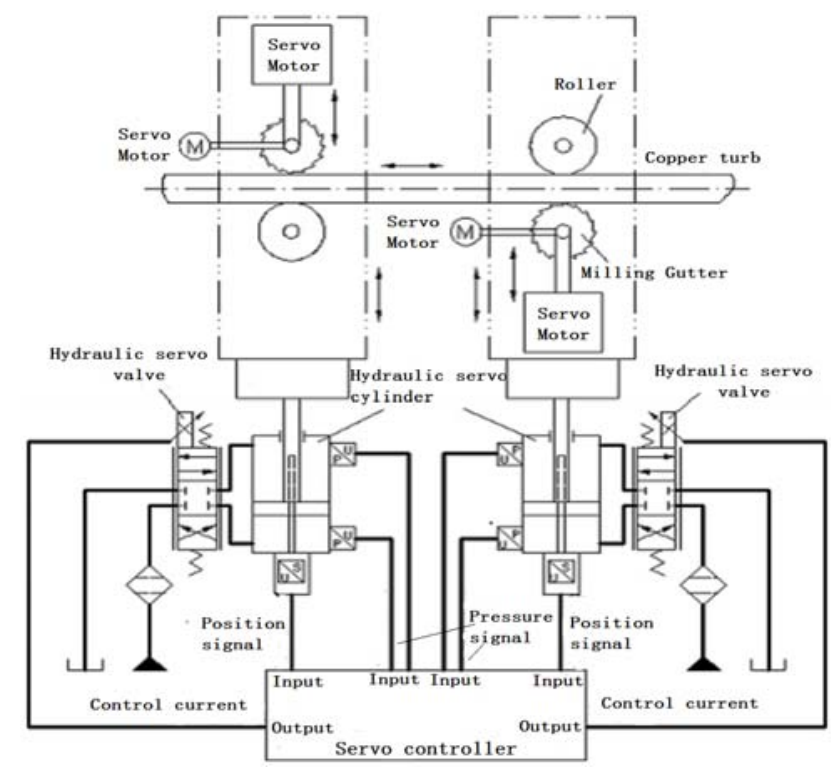

Figure 1. Dynamic mechanism and its control system of milling machine milling diagram

For the milling cutter and roller follow the curve of the copper pipe all the time, the electro-hydraulic servo system carries out the position control of milling power by control the movement of the servo cylinder piston[2]. By sampling on the servo cylinders, pressure and position signal, Servo controllers control the core processing and output current or voltage driven servo valve after the operation, and thereby control the input oil flow to the realization of servo cylinder servo position control of milling power. 


\section{SYSTEM HARDWARE DESIGN}

According to the basic requirements of peripheral devices and control system of electro-hydraulic servo system, the system should have excellent characteristics such as high control precision, fast speed, and adaptability of control strategies. According to the requirements of the control system of milling machine, the controller hardware system design as shown in Figure 2, the whole system consists of core module, signal processing module, servo-driven modules, human-computer interaction modules and other components.

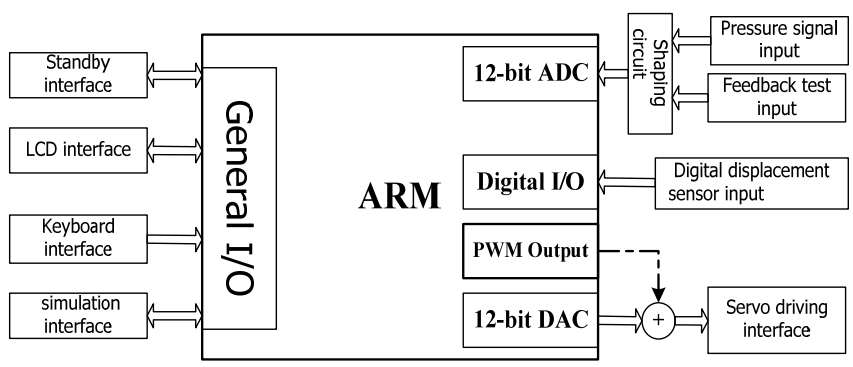

Figure 2. controller hardware system

1) Core processing module: the core processing module adopts STM32F107RCT6 which is based on 32-bit flash microcontroller with ARMCortex-M3 kernel, as several improvements have been done on the structure of Cortex-M3 for this series of chips, which improve the performances and reduce the energy consumption simultaneously. The STM32 has a maximum operating frequency at $72 \mathrm{MHz}$ and a data handling capacity of $1.25 \mathrm{DMIPS} / \mathrm{MHz}$, which not only excellently completes digital logic operation, improve the flexibility of control systems, but also enriches the on-chip peripherals, and simplifies the traditional analog circuits, finally increases the integration of system which can meet both requirements of real-time and low energy consumption.

2) Signal acquisition module: the signals need to be collected by position servo controller of milling machine includes: up and down chamber pressures of the servo cylinder, servo cylinder piston rod position and servo valve feedback current[3]. Through STM32 single chip microcontroller embedded a 12-bit ADC module,data is extracted.In order to solve the inconsistency between the sensor signal return and the reference voltage of single chip microcontroller ADC12 module,the voltage conversion is completed by means of resistor divider, which can meet the requirements for voltage sampling of $A / D$ converter. To prevent the input signal abnormalities or anthropogenic signal line fault which will cause the voltage is so excessively high that ADC12 module even STM32 chip may be bured down, BAT54S as the voltage protector is needed. Finally, the high-frequency interference is filtered out by the hardware RC low pass filter circuit, which can improve the stability of the system.In feedback circuit of servo valve, $-40 \sim 40 \mathrm{~mA}$ for current feedback, input sampling standards of $0 \sim 2.5 \mathrm{~V}$ for ADC12 module. Therefore, current signal needs to be converted into linear related voltage signal first, then negative voltage needs to be converted into positive. System uses a $-1.25 \mathrm{~V}$ bias voltage, drop resistance and multiple-input operational amplifier combination to complete the functionality described above.According to the analysis of output circuit of control signal, voltage value range of UCRNT should be less than $-1.25 \sim+1.25 \mathrm{~V}$. Then, with basic knowledge of the operational amplifier circuit, the relationship between output voltage UVLTG and voltage UCRNT can be derived as:

$$
U_{V L T G}=-\left(\frac{R_{f}}{R_{1}} \cdot(-1.25)+\frac{R_{f}}{R_{2}} U_{C R N T}\right)
$$

ADC12 module signal sampling standards can be meet by matching resistors. Schematic diagram of intermediate conversion of the current signal acquisition circuit is shown in Figure 3.

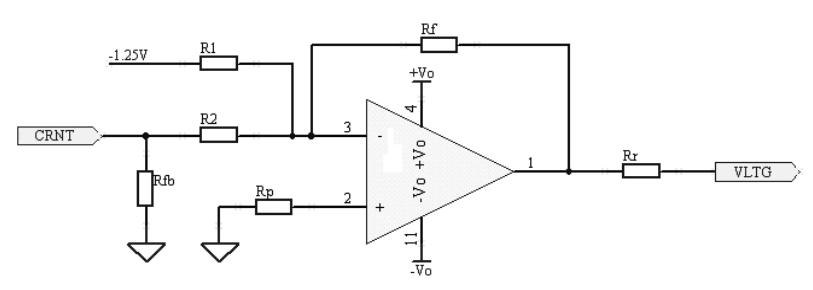

Figure 3. current signal conversion circuit

3) Output module control: the direct control object of milling machine servo is the servo-valve. By means of power amplifiers not only the control power is amplified, but also the switch between voltage, and current and the switch between unipolar control signal and bipolar control signal are realized.

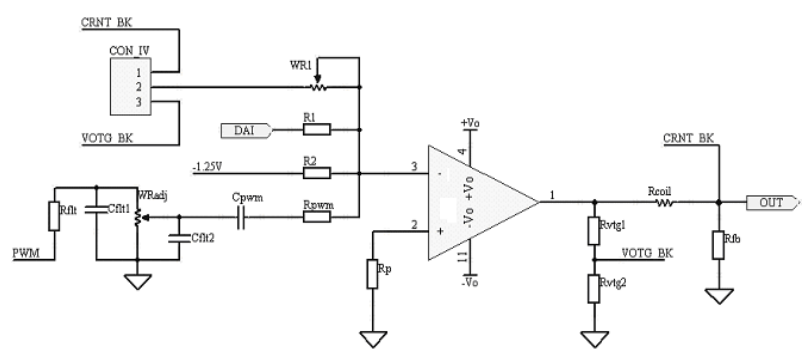

Figure 4. control output circuit principle schematic diagram

50\% duty PWM square wave output is achieved using single-chip microcomputer built-in timers and output capture modules. After second-order filter, overlying the square wave on the control signals to achieve approximate sinusoidal signal characteristics of servo valve chatter, which reduces the hysteresis of servo valve and providing flexibility of servo valve letter action.Control output circuit schematic diagram shown in Figure 4.Finally, grounding techniques, isolation technology and modular layout are adopted to control the reliability of the system hardware effectively, which leads to guarantee the stability of the system in a complex industrial field[4]. 


\section{SYSTEM CONTROL STRATEGY AND SOFTWARE DESIGN}

In industrial control applications, specially with real-time requirements, the program must be task deployed. Position servo controller of milling machine needs to real-time control of servo cylinder location and movement.Therefore, the control system adopts a foreground and background system for real-time task deployments. The structure of system software is modular programming to strengthen the reliability and maintainability of software.The overall structure of the system software was divided into control method of data acquisition and processing module, system modules, system control and output module liquid crystal display and keyboard module.
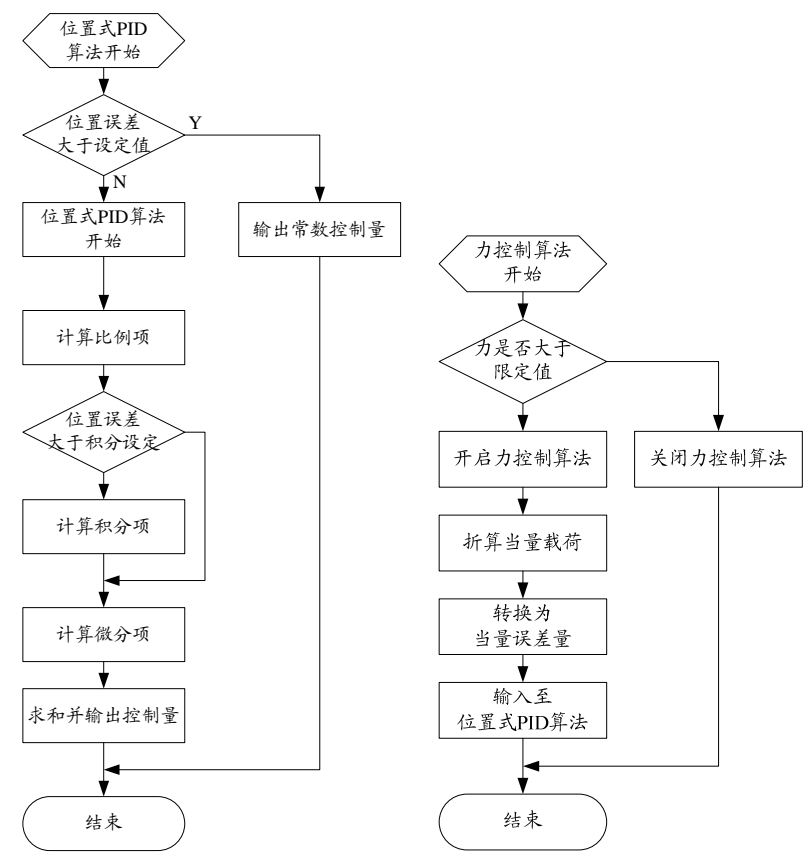

Figure 5. Position control and force control algorithm flowchart

To meet the basic requirements of compensate position and force control of hydraulic cylinder position, position control and force control algorithm is designed. Position control algorithm plays the role of servo control of servo cylinder piston rod position.Equivalent error is treated as input in the algorithm, through the proportional component, differential and integral operations, and sum to achieve the ultimate control which can drive DAC output[5]. The aim of servo force control algorithm is to convert additional load on the system converted into PID error entry. By simulating the output directive, the position compensation for force control can be completed. In addition, position control hysteresis loop characteristics result from the algorithm can also get compensation by improving algorithm. Position control and force control algorithm flowchart as shown in Figure 5.

\section{SYSTEM TESTING AND EXPERIMENTAL VERIFICATION}

To verify the performance of position servo controller of milling machine developed, simulation experiments are taken in lab. Plunger pump of hydraulic system is adopted as hydraulic power source, through overflow valve adjustment system of three concentric axis pressure enters the servo valve. Fluid flow and fluid flow direction is controlled by servo valves, in and out of servo cylinder after rod and Rodlumen, return oil flow by the servo valve back to the fuel tank.Schematic of experiment platform as shown in Figure 6.

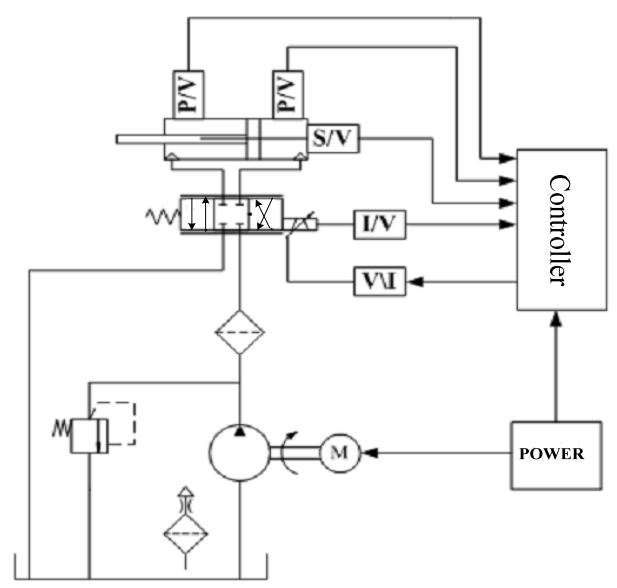

Figure 6. experimental platform schematic

The system at pressure 4Mpa of step signal, traffic to $15 \mathrm{~L} / \mathrm{min}$, 25mm condition, System PID parameter is set to $\mathrm{Kp}=15.0, \mathrm{Ki}=5.0, \mathrm{Kd}=10.0$ testing control system for timedomain response curve as shown in Figure 7.

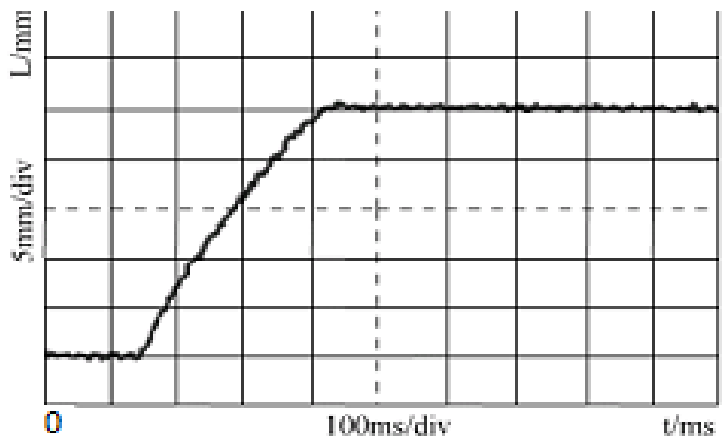

Figure 7 .Time-domain response curve of control system

Tests can be seen, the system power on the rise time of the transient process of $250 \mathrm{~ms}$, without overshoot.Use indicator and oscilloscope measurement system of steadystate control accuracy of $\pm 0.030 \mathrm{~mm}$, single axis servo for $300 \mu$ s.Servo cylinder motion smoothing smooth, no oil leakage phenomenon.Experimental data indicates that the system is stable, reliable, in addition to guarantee controlling precision reached fast running speed, meet the requirements of the processing of milling machines. 


\section{CONCLUSION}

Electro-hydraulic servo control system with deep integration of electronic control technology and hydraulic drive technology is adopted, which enables the system not only gets characteristics of fast and flexible as electronic control system does, but also capabilities of overload and stability as hydraulic system does. The ARM core adopted can keep running with high speed and avoiding too much peripheral expansion with its wealthy on-chip resources, which simultaneously shortens the servo cycle and improves the integration of system. Both the control characteristics and the position precision of system are improved by adopts composite control algorithm of position and force control. The problems of domestic control system such as: low control accuracy, slow speed, poor stability and so on can be well solved by this system. With keeping the copper surface oxide thickness uniformly during milling, as well as reducing copper loss and improving productivity, this system is promising on engineering application.

\section{REFERENCES}

[1] Runhua Tan, Jibing Sun and Qingsuo Liu: Advanced Materials Research, Vol. 542-543(2012),p.563-566.

[2] C.H.Lv,L.P.Xu,D.Z. Ren and L.L. He: Chinse Hydraulics\&Pneumatics, Vol.5(2008), p.24. (In Chinese)

[3] T.H. Sun, C.C. Tseng and M.S. Chen: Chinese Journal of Aeronautics, Vol. 21 (2008) No.4, p.378-384.

[4] C.J.Ru,B.Jing,G.S.Wu:ComputerEngineering\&Sinence, Vol.34 (2012) No.1, p.154. (In Chinese)

[5] W.J.Liu,F.Y.Zhou,W.G.Pan:SHIP ENGINEERING, Vol.6(2009), p.56. (In Chinese) 